\title{
Material Characterization and Thermal Performance of Au Alloys in a Thin-Film Plasmonic Waveguide
}

\author{
F. Bello ${ }^{1,2,3}$, O. K. Orhan ${ }^{1}$, N. Abadía ${ }^{4}$, D. D. O’Regan ${ }^{1}$, and J. F. Donegan ${ }^{1,2,3}$

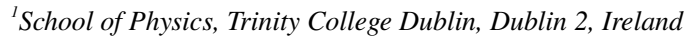 \\ ${ }^{2}$ Centre for Research on Adaptive Nanostructures and Nanodevices (CRANN), Trinity College Dublin, Dublin 2, Ireland \\ ${ }^{3}$ AMBER Research Centre, Trinity College Dublin, Dublin 2, Ireland \\ ${ }^{4}$ School of Physics and Astronomy, Institute for Compound Semiconductors, Cardiff University, Cardiff CF24 3AA, U.K. \\ fbello@tcd.ie
}

\begin{abstract}
We investigate heatsinking methods and material properties of various Au alloys to be used within thin-film plasmonic resonators to create optimal heating conditions in near-field transducers, with demonstrated application towards heat-assisted magnetic recording devices. ${ }^{1}$ OCIS codes: $160.0160,310.6845$.
\end{abstract}

\section{Conceptual Design}

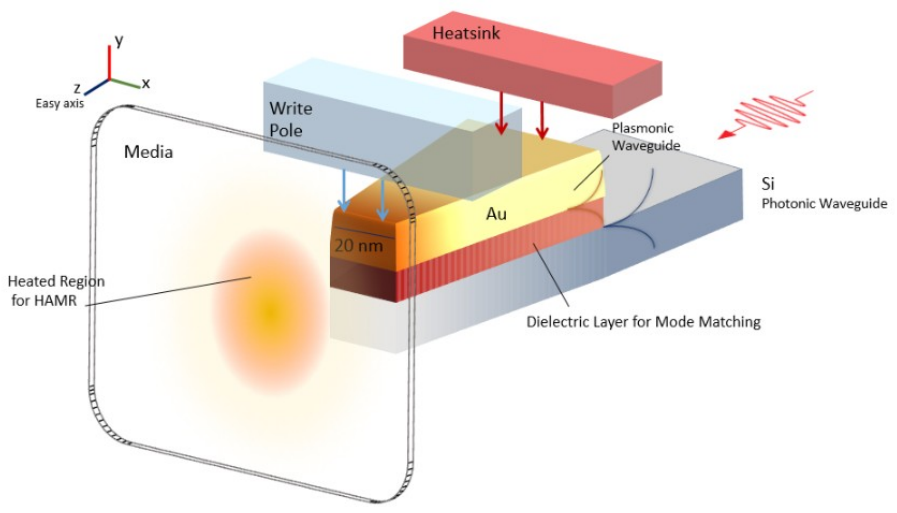

Fig. 1. Schematic of the hybrid plasmonic waveguide used as a near-field light source in order to heat nearby magnetic media above its Curie temperature for heat-assisted magnetic recording (HAMR). Thermal performance within the media as well as stability of the metallic components is analyzed as a function of heatsink placement and alloying of the metallic layer.

The fabrication of ultrathin plasmonic resonators, that are a few to tens of nanometers in thickness, is now required by industry for a number of applications ranging from quantum information processing [1] to magnetic recording [2]. The latter in particular demands control of not only the optoelectronic performance, but also heat diffusive properties within the resonator and nearby media. We examine the caloritronics, i.e. the generation of heat currents, in plasmonic near-field transducers (NFT) along with thermal gradients.

We focus on a NFT previously optimized for use within heat-assisted magnetic recording, or HAMR, devices using $\mathrm{Au}$ thin films and heat sinking components [2]. For HAMR, we are required to achieve temperatures above the Curie value within the magnetic media while keeping thermal and mechanical stability of a NFT roughly $10 \mathrm{~nm}$ from this region. A large impediment to the lifetime of the devices is related to the temperature within the metallic components of the HAMR apparatus, i.e. Gold, which is often used in resonators for its superior plasmonic performance and the write pole sitting above this layer (See schematic of NFT in Fig. 1). We investigate how to manipulate heatsinking components while causing minimal change to heating in the media. This includes looking at the effects on the optical and thermal performance when using various Au-alloys within the NFT. Au may deform due to resistive losses as it lowers its energy state at elevated temperatures. Thus Au alloys are sought after which must produce the required heat currents, typically on the order $10^{11} \mathrm{~W} / \mathrm{m}^{2}$, in order to create sufficient temperature gradients $(10-20 \mathrm{~K} / \mathrm{nm})$ for state-of-the-art areal densities and bit writing [2].

\section{Results}

1The authors acknowledge funding from Marie Skłodowska-Curie grant 713567, AMBER 12/RC/2278 and 15/IA/2854, EPSRC EP/P006973/1 
In order to calculate material parameters such as thermal conductivity shown in Table 1, starting geometries for

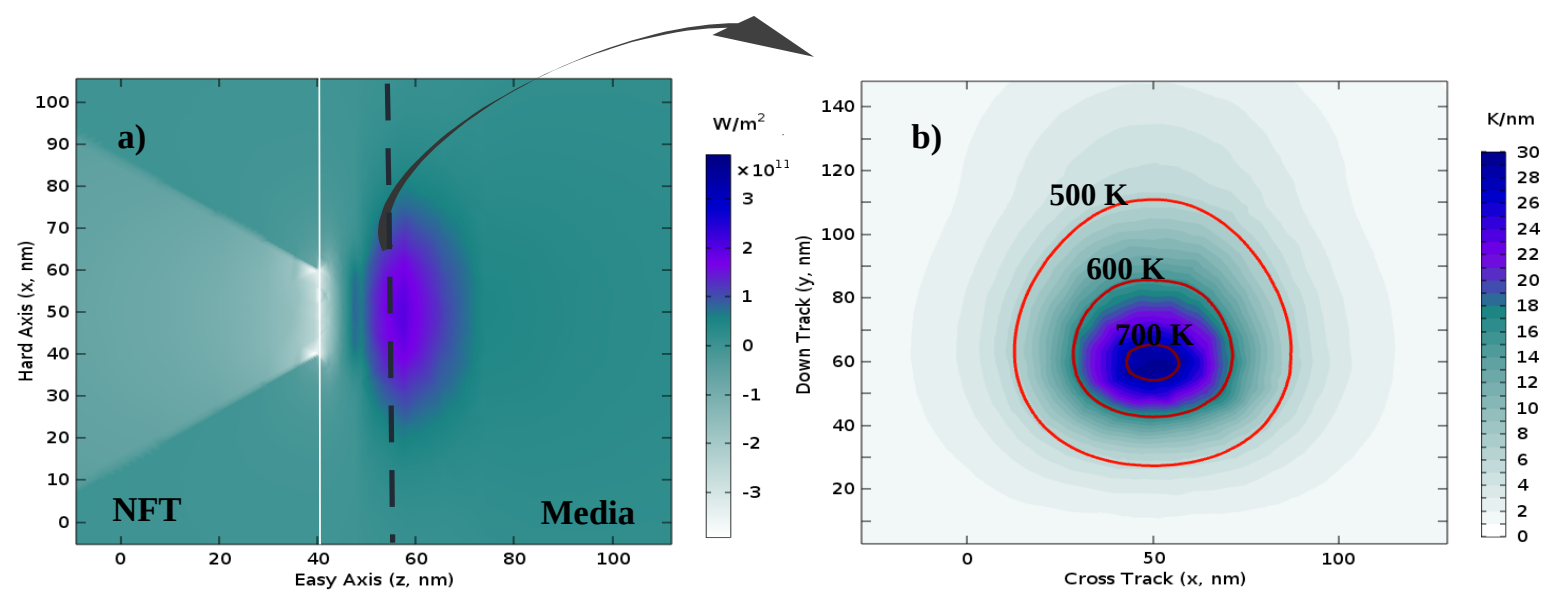

Fig. 2. a) Deposition plane view at the Au-dielectric interface showing heat currents on the order of $1011 \mathrm{~W} / \mathrm{m} 2$ along the easy axis direction. Heat currents on this order are anticipated to produce thermal gradients are the order of $101 \mathrm{~K} / \mathrm{nm}$ [3]. b) Thermal gradients (magnitude) along the hard axis of the magnetic recording layer (black dashed line) and temperature contours (red) demonstrating the ability to reach above the Curie temperature $(\approx 750 \mathrm{~K})$. Maximum temperature in the Au for the presented case is $403 \mathrm{~K}$.

ordered alloys were constructed using super cells of pure Ag or Pd and substituting the desired number of Au atoms to achieve the primitive unit cells. Geometry optimizations, self-consistent field and non-self-consistent field simulations were performed with the Quantum ESPRESSO software [4]. The Fermi velocities were evaluated starting from the Kohn-Sham (KS) band-structures for each alloy. Stretching operators, which were approximated by using the one-shot non-self-consistent GW method, were used to improve the descriptions of the underlying KS band-structures in spectroscopy simulations. The density of states at the Fermi level as well as contributions of inter-band transitions to optical spectra were calculated using the random-phase approximation starting from the stretched KS band-structures within the Yambo code [5]. Material parameters were then used within finite-element simulations to retrieve the steady state solutions of the Helmholtz and heat diffusion equations that are presented in Fig. 2 [6].

Table 1. Thermal Conductivity (W/mK) Au-Ag Alloys

\begin{tabular}{cccccc}
\hline $\mathrm{Au}$ & $\mathrm{AuAg}_{7}$ & $\mathrm{AuAg}_{3}$ & $\mathrm{AuAg}$ & $\mathrm{Au}_{3} \mathrm{Ag}$ & $\mathrm{Au}_{7} \mathrm{Ag}$ \\
\hline 319 & 355 & 681 & 475 & 612 & 277 \\
\hline
\end{tabular}

Sample results for pure Au are shown in Fig. 2a, where we have calculated the heat currents ( $\propto \nabla T / \nabla z)$ generated at the Au-dielectric interface of the NFT. Values of roughly $10^{11} \mathrm{~W} / \mathrm{m}^{2}$ are produced by the NFT over a region of a few tens of nanometers. We thus are able to match state-of-the-art values previously reported for thermal gradients in systems undergoing similar heat currents [3]. The currents are also able to produce temperatures well above the Curie temperature; however, issues arise in keeping the Au close to ambient temperatures. This is particularly due to movement and heated air/lube between the media and NFT. All media parameters and material properties are reported in [2]. We demonstrate improvements in thermal conductivities using Au-Ag and Au-Pd alloys, and perform a comparative analysis of the thermal performance when using only $\mathrm{Au}$. In particular, we demonstrate the effects on generating thermal gradients within the nearby magnetic media as shown in Fig. $2 \mathrm{~b}$ while maintaining thermal stability of the NFT.

\section{References}

[1] H. Groß, J. M. Hamm, T. Tufarelli, O. Hess, and B. Hecht, “Near-field strong coupling of single quantum dots,” Sci. Adv. 4, eaar4906 (2018).

[2] N. Abadía et al, "Optical and thermal analysis of the light-heat conversion process employing an antenna-based hybrid plasmonic waveguide for HAMR," Opt. Express 26, 1752-1765 (2018).

[3] R. St-Gelais, L. Zhu, S. Fan, and M. Lipson, "Near-field radiative heat transfer between parallel structures in the deep subwavelength regime,” Nature Nanotech. 11, 515-519 (2016).

[4] P. Giannozzi et al, “Advanced capabilities for materials modelling with Quantum ESPRESSO,” J. Phys.: Condens. Matter 29, 465901 (2017).

[5] A. Marini et al, "Yambo: an ab initio tool for excited state calculations," Comput. Phys. Commun. 180,1392 - 1403 (2009).

[6] COMSOL Multiphysics ${ }^{\mathrm{TM}}$ v.5.3a www.comsol.com. COMSOL AB, Stockholm, Sweden. 